\title{
Ultrasound Study File
}

National Cancer Institute

\section{Source}

National Cancer Institute. Ultrasound Study File. NCI Thesaurus. Code C115503.

A collection of ultrasound image files pertaining to a clinical study. 